\title{
ON THE HOMOTOPY THEORY OF SIMPLICIAL LIE ALGEBRAS
}

\begin{abstract}
STEWART B. PRIDDY
Abstract. Elements $\lambda_{n}, n \geqq 0$, which generate the homotopy groups of spheres in the category of simplicial Lie algebras are shown to have Hopf invariant one. This fact is shown to have strong implications for the homotopy theory of this category.
\end{abstract}

In 1958, Kan [5] constructed an algebraic model (simplicial groups) for homotopy theory. Since then, various group theoretic methods have been used to study this model. In 1965, Curtis [3] showed that the lower central series filtration of a group induces a spectral sequence for computing homotopy groups of a simplicial group. This spectral sequence starts with the homotopy groups of a simplicial Lie algebra. In this sense the homotopy theory of simplicial Lie algebras is a first approximation to ordinary homotopy theory.

The purpose of this note is to describe this approximation from the point of view of an appropriately defined analogue of the Hopf invariant. We shall be concerned with the following statements which reveal something of the simplicity of the homotopy structure of simplicial Lie algebras.

A. There are elements of Hopf invariant one for every integer $n \geqq 0$.

B. The Steenrod algebra is bigraded with $S q^{i}$ having bidegree $(i, 1)$ for $i>0 ; S q^{0}$ is identically zero.

C. The Adams spectral sequence for spheres collapses $\left(E^{2}=E^{\infty}\right)$.

$D$. The homotopy groups of spheres are generated by elements of Hopf invariant one under composition.

E. The EHP sequence is short exact.

This note is intended as an epilogue to [1] in which a stable mod $p$ version of the Curtis spectral sequence yielding a new $\left(E^{1}, d^{1}\right)$-term of the Adams spectral sequence is studied. ${ }^{1}$ Results $\mathrm{A}-\mathrm{E}$ are due to the authors of [1] and the present author. All have appeared previously [1], [9], [4], with the exception of $A$, which was first noted by $\mathrm{D}$. Quillen. We hope, however, that the general homotopy theorist will find the Hopf invariant theme used here more intuitive.

Received by the editors July 30, 1969.

AMS Subject Classifications. Primary 5540, 5534.

Key Words and Phrases. Simplicial Lie algebras, Hopf invariant, Adams spectral sequence, Steenrod algebra, suspension sequence.

1 For a homological description see [10]. 
1. Preliminaries. We assume some knowledge of the homotopy theory of simplicial sets and groups (e.g., May's book [6]).

Let $s \&$ denote the category of simplicial restricted Lie algebras $\$$ over $\boldsymbol{Z}_{2}$ (see $[7, \S 6]$ for restricted). Homotopy groups $\pi_{*}$ (5) are obtained by viewing (\$) as a chain complex with differential $\partial_{n}=\sum_{i=0}^{n}(-1)^{i} d_{i}$, thus

$$
\left.\pi_{n}(S)=H_{n}(\$) ; \partial_{*}\right)=\operatorname{ker} \partial_{n} / \operatorname{im} \partial_{n+1} .
$$

By the $n$-sphere of $s \&$ we mean $L A S_{n}$, where $S_{n}$ is the simplicial set sphere, $A$ is the free $\boldsymbol{Z}_{2}$-module functor with the basepoint set equal to zero, and $L=\sum_{r \gtrless 1} L_{r}$ is the free restricted Lie algebra functor (see $[11,2.3])$. With the appropriate notion of Lie homotopy, the groups $\pi_{*}(B)$ are just the Lie homotopy classes of maps $L A S_{n} \rightarrow(B)$ of $s \&$.

Homology and cohomology groups for $s \mathfrak{L}$ are given by

$$
H_{0}(\$)=0, \quad H_{*}(\$)=\pi_{*} \bar{W} U\left(\xi, \quad H^{*}(\xi)=\operatorname{Hom}\left(H_{*}\left(\$, Z_{2}\right)\right.\right.
$$

where $\bar{W}$ is the Eilenberg-MacLane functor and $U$ is the universal enveloping algebra functor $[9, \S \S 2.2-2.3]$. As a functor $H^{n+1}$ is represented by an Eilenberg-MacLane complex $K\left(\boldsymbol{Z}_{2}, n\right)$ for $s \mathscr{Q}$ [9, 3.13.3]. Cup products are obtained from the natural diagonal map $U(S) U(S) U S$ of universal enveloping algebras (see $[9, \S \S 2-3]$ for a more complete discussion of (co-) homology).

If $(B)$ is a free simplicial Lie algebra (i.e. $\$_{n}=L M_{n}$ for some $Z_{2}$-module $M_{n}$, and $\left.s_{i} M_{n} \subset M_{n+1}\right)$ then an alternate description is

$$
\left.H_{*}(\mathrm{~S})=\pi_{*-1} \mathrm{Ab} \text { ( }\right), \quad * \geqq 1
$$

where $\mathrm{Ab}$ is the abelianization functor. Thus, in particular, for the sphere object $L A S_{n}$

$$
H_{*} L A S_{n}=\pi_{*-1} A S_{n}=Z_{2} .
$$

2. The Hopf invariant. In this section we show that the maps $\lambda_{n}$ of $[1,5.2]$ have Hopf invariant one. To define this notion for simplicial Lie algebras we follow Steenrod $\left[12\right.$, p. 12]. Given a map $\alpha: L A S_{2 n}$ $\rightarrow L A S_{n}$ of $s \mathfrak{L}(n \geqq 0)$ we "attach a cell" by $\alpha$ forming a new simplicial Lie algebra $L A S_{n} \cup_{\alpha} Z_{2 n+1}$ where

$$
\begin{aligned}
\left(L A S_{n} \cup_{\alpha} Z_{2 n+1}\right)_{i} & =L\left(A S_{n}\right)_{i}, \quad 0 \leqq i \leqq 2 n \\
& =L\left(\left(A S_{n}\right)_{2 n+1} \oplus Z_{2}\left(Z_{2 n+1}\right)\right), \quad i=2 n+1 \\
& =L\left(\left(A S_{n}\right)_{i} \oplus Z_{2}\left(i \text {-dim. degeneracies of } Z_{2 n+1}\right)\right), \\
&
\end{aligned}
$$

The face and degeneracy operators of $L A S_{n} \cup_{\alpha} Z_{2 n+1}$ are determined 
by those of $L A S_{n}$ and by setting $d_{0} Z_{2 n+1}=\alpha\left(i_{2 n}\right), d_{i} Z_{2 n+1}=0, i>0$. To exclude the possibility of attaching a cell to $i_{n}$ we require that $\alpha\left(i_{2 n}\right)$ $\in \sum_{r \geq 2} L_{r} A S_{n}$, in view of this:

LEMMA.

$$
\begin{aligned}
& H^{i}\left(L A S_{n} \cup_{\alpha} Z_{2 n+1}\right)=Z_{2}, \quad i=n+1, \quad 2 n+2, \\
& =0, \quad \text { otherwise. }
\end{aligned}
$$

PRoOF. Since $L A S_{n} \cup_{\alpha} Z_{2 n+1}$ is free it follows that

$$
H_{*}\left(L A S_{n} \cup_{\alpha} Z_{2 n+1}\right)=\pi_{*-1} \mathrm{Ab}\left(L A S_{n} \cup_{\alpha} Z_{2 n+1}\right) .
$$

But $\alpha\left(i_{n}\right) \in \sum_{r \geq 2} L_{r} A S_{n}$ implies that $\mathrm{Ab}\left(L A S_{n} \cup_{\alpha} Z_{2 n+1}\right)=A S_{n}$ $\oplus A S_{2 n+1}$ which are Eilenberg-MacLane complexes. Hence $H^{*}\left(L A S_{n} \cup_{\alpha} Z_{2 n+1}\right)=\operatorname{Hom}\left(H_{*}\left(L A S_{n} \cup_{\alpha} Z_{2 n+1}\right), Z_{2}\right)=Z_{2}+Z_{2}$ as asserted.

DEFinition. If $h_{n+1}$ and $h_{2 n+2}$ are cocycles representing the two nonzero classes of $H^{*}\left(L A S_{n} \cup_{\alpha} Z_{2 n+1}\right)$ then $\left[h_{n+1}\right] \cup\left[h_{n+1}\right]=H(\alpha)\left[h_{2 n+2}\right]$ where $H(\alpha) \in Z_{2}$. Now $H(\alpha)$ depends only on the Lie homotopy class of $\alpha$ and is called the Hopf invariant of $\alpha$.

Let $\lambda_{n}: L A S_{2 n} \rightarrow L A S_{n}$ be the map (see $[1,5.2]$ ) of simplicial Lie algebras defined by $i_{2 n} \rightarrow \sum s_{\alpha_{n}} \cdots s_{\alpha_{1}} i_{n} \otimes s_{\beta_{n}} \cdots s_{\beta_{1}} i_{n}$ where the sum is taken over all $(n, n)$ shuffles of $(0,1, \cdots, 2 n-1)$. If $n=0, \lambda_{0}$ is defined by $i_{0} \rightarrow i_{0} \otimes i_{0}$. For $n \geqq 0$, let $i_{n} \triangleq i_{\text {* }}$ denote these shuffle products.

Theorem. For $n \geqq 0, H\left(\lambda_{n}\right)=1$.

Proof. Let $M=L A S_{n} \cup_{\lambda_{n}} Z_{2 n+1}$. In order to compute $\left[h_{n+1}\right]$ $\cup\left[h_{n+1}\right]$ we use the bar construction $\bar{B} N U M$ (where $N$ is the normalization functor) instead of $\bar{W} U M$. Recall [8, Theorem 1] that there is a homology equivalence $g: \bar{B} N U M \rightarrow N \bar{W} U M$. It can be shown that $\mathcal{G}$ is a map of differential coalgebras.

Consider the chain $c=\left[Z_{2 n+1}\right]+\left[i_{n} \mid i_{n}\right] \in \bar{B}_{2 n+2} N U M$. Now $\partial c$ $=\partial\left[Z_{2 n+1}\right]+\partial\left[i_{n} \mid i_{n}\right]=i_{n} \stackrel{\otimes}{=} i_{n}+i_{n} \stackrel{\otimes}{i_{n}}=0$ and so the cocycle $h_{n+1} \cup h_{n+1}$ is not a coboundary since

$$
\begin{aligned}
\left(h_{n+1} \cup h_{n+1}\right)(c) & =\left(h_{n+1} \otimes h_{n+1}\right) \Delta c \\
& =\left(h_{n+1} \otimes h_{n+1}\right)\left(\left[i_{n}\right] \otimes\left[i_{n}\right]\right)=1 \otimes 1=1 .
\end{aligned}
$$

Hence $h_{n+1} \cup h_{n+1}=h_{2 n+2}$.

3. Implications of the Hopf invariant. Topologically, it is well known that a map $S^{2 n-1} \rightarrow S^{n}$ has Hopf invariant one only if $n=2^{k}$. This follows from the Adem relations which imply that $S q^{n}$ is indecomposable if and only if $n=2^{k}$. 
The situation for $s \mathscr{L}$ is quite different. In $[9, \S 5]$ we showed that Steenrod squares, $S q^{i}, i \geqq 0$, could be defined for $s \&$. These operations automatically satisfy the usual axioms and Adem relations except that $S q^{0}$ is not necessarily the identity. It follows from representability $[9, \S 3]$ that $S q^{0}$ is either the identity or zero. However, the existence of the Hopf invariant one elements $\lambda_{n}$ shows that $S q^{n+1}$ is indecomposable for all $n \geqq 0$, thus $S q^{0} \neq 1$, and so $S q^{0}=0$. Thus for example, the usual relation $S q^{1} S q^{2}=S q^{3} S q^{0}=S q^{3}$ holding for the category of topological spaces now becomes $S q^{1} S q^{2}=S q^{3} S q^{0}=0$ for $s \&$.

The existence of these Hopf invariant one elements also has a drastic effect on the homotopy groups $\pi_{*} L A S$ of the sphere spectrum for $s \mathscr{L}$. In $[9,9.1]$ we showed that there is an Adams spectral sequence

$$
E_{*, *}^{2}=\operatorname{Ext}_{\mathscr{L}}^{*, *}\left(\boldsymbol{Z}_{2}, \boldsymbol{Z}_{2}\right) \Rightarrow \pi_{*} L A S
$$

where $\mathfrak{A}_{\mathfrak{L}}$ is the algebra of Steenrod operations for $s \mathfrak{L}$. Direct computation shows $E_{*, *}^{2}$ to be an algebra generated by the elements $\xi_{n}$ $\in \operatorname{Ext}_{\mathfrak{A} \mathcal{L}}^{1, n}$ (where $\xi_{n}$ is dual to $S q^{n}$ in the admissible basis $[9,7.3]$ ). Since $H\left(\lambda_{n}\right)=1, \xi_{n+1}$ persists to $\lambda_{n}$ and $E^{2} \approx E^{\infty}$. Hence $\pi_{*} L A S$ is generated by the $\lambda_{n}$ 's under composition. This contrasts with the topological situation where J. Cohen [2] has shown that the 2-primary component of the homotopy group $\pi_{*}(S)$ of the (geometric) sphere spectrum is generated by the Hopf invariant one classes $\eta, \nu$, and $\sigma$ under composition and higher Toda brackets.

The Hopf invariant also plays a significant role with respect to the unstable homotopy groups $\pi_{*} L A S_{n}$ of spheres for $s \mathcal{L}$. These groups are related by a short exact EHP (or Whitehead-James) sequence

$$
0 \rightarrow \pi_{*-1} L A S_{n-1} \stackrel{E}{\rightarrow} \pi_{*} L A S_{n} \stackrel{H}{\rightarrow} \pi_{*} L A S_{2 n} \rightarrow 0
$$

where $E$ is the suspension map and $H$ is the Hopf invariant map. These maps are defined on basis elements by

$$
\begin{aligned}
E\left(\lambda_{i_{1}} \cdots \lambda_{i_{r}} 1_{n-1}\right) & =\lambda_{i_{1}} \cdots \lambda_{i_{r}} 1_{n}, \\
H\left(\lambda_{i_{1}} \cdots \lambda_{i_{r}} 1_{n}\right) & =\lambda_{i_{2}} \cdots \lambda_{i_{r}} 1_{2 n} \quad \text { if } i_{1}=n, \\
& =0 \quad \text { otherwise. }
\end{aligned}
$$

Recall that $\pi_{*} L A S_{n}$ has a $\boldsymbol{Z}_{2}$-module basis consisting of all monomials $\lambda_{i_{1}} \cdots \lambda_{i_{r}} 1_{n}, i_{1} \leqq n, i_{j+1} \leqq 2 i_{j}$. A split version of this sequence was used in $[1,5.6]$ to compute $\pi_{*} L A S_{n}$ inductively for all $n$. In [4] Curtis points out that $E$ and $H$ are maps of differential $\boldsymbol{Z}_{2}$-modules and uses this sequence to establish some nonzero homotopy groups of spheres. 


\section{REFERENCES}

1. A. K. Bousfield, E. B. Curtis, D. M. Kan, D. G. Quillen, D. L. Rector and J. W. Schlesinger, The mod-p lower central series and the Adams spectral sequence, Topology 5 (1966), 331-342. MR 33 \#8002.

2. J. M. Cohen, The decomposition of stable homotopy, Ann. of Math. (2) 87 (1968), 305-320. MR 37 \#6932.

3. E. B. Curtis, Some relations between homotopy and homology, Ann. of Math. (2) 82 (1965), 386-413. MR 32 \#1704.

4. - Some nonzero homotopy groups of spheres, Bull. Amer. Math. Soc. 75 (1969), 541-544.

5. D. M. Kan, On homotopy theory and c.s.s. groups, Ann. of Math. (2) 68 (1958), 38-53. MR 22 \#1898.

6. J. P. May, Simplicial objects in algebraic topology, Van Nostrand Math. Studies, no. 11, Van Nostrand, Princeton, N. J., 1967. MR 36 \#5942.

7. J. Milnor and J. C. Moore, On the structure of Hopf algebras, Ann. of Math. (2) 81 (1965), 211-264. MR 30 \#4259.

8. J. C. Moore, Comparison de la bar construction a la construction $W$ et aux complexes $K(\pi, n)$, Séminaire H. Cartan (1954/55) exposé 13, Secrétariat mathématique, Paris, 1955. MR 19, 438.

9. S. B. Priddy, Primary cohomology operations for simplicial Lie algebras, Illinois J. Math. (to appear).

10. - Koszul resolutions, Trans. Amer. Math. Soc. (to appear).

11. D. L. Rector, An unstable Adams spectral sequence, Topology 5 (1966), 343346. MR 33 \#8003.

12. N. E. Steenrod and D. B. A. Epstein, Cohomology operations, Ann. of Math. Studies, no. 50, Princeton Univ. Press, Princeton, N. J., 1962. MR 26 \#3056.

NorthWESTERn University, Evanston, ILLINOIS 60201 